\title{
E-Learning Assistant System Based on Virtual Human Interaction Technology
}

\author{
Xue Weimin $^{1,2}$ and Xia Wenhong ${ }^{1}$ \\ ${ }^{1}$ Institute of Information Technology, Beijing Union University, Beijing 100101 \\ ${ }^{2}$ Department of Computer Science \& Technology, Tsinghua University, Beijing 100084 \\ xuewmemail.tsinghua.edu.cn
}

\begin{abstract}
The virtual human affective interaction is one of the hotspots in information science and cognitive science today. The importance of emotions in learning process is more and more acknowledged. This paper introduces a new virtual human interaction module based on multi-agents. The affective interactive model is built according to the human cerebrum control pattern. The multimodal detection agents are able to help tutor to better understand the emotional and motivational state of the learner throughout the learning process. The results of the practical application virtual human interaction in E-Learning system indicate that virtual human technology can improve the interactivity and entertainment for the E-learners.
\end{abstract}

Keywords: E-Learning, virtual human, multi-agents model.

\section{Introduction}

The virtual human with affection is challenging project facing to modern scientists, especially when those characters interact with real people possessing real affection. In biological systems, the existence of emotions can have both advantageous and detrimental effects on the cognitive process [1]. Now a growing amount of studies support the claim that affection plays a critical role in decision-making and learning performance as it influences cognitive processes [2]. For example, as suggested by Goleman [3] "the extent to which emotional upsets can interfere with mental life is no news to teachers. Students who are anxious, angry or depressed don't learn; people who are caught in these states do not take information efficiently or deal with it very well'. So it is obviously that the user's affective state plays an important role in improving the effectiveness of e-learning. The emotional unawareness has been considered one of the main limits of the traditional e-learning tools. In fact, while skilled teachers can modify the learning path and their teaching style according to the feedback signals provided by the learners, e-learning platforms cannot generally take account of these feedbacks resulting often too rigid and weakened [4].This paper presents our studies on affective interaction between real human and virtual human. The virtual human can perceive real human affection by recognition of his voices and facial expressions, and the virtual human can generate his simple affective behavior autonomously [5]. 


\section{The Virtual Human Affective Interaction Theory}

\subsection{The Characters of Virtual Human}

Affective virtual human is to make virtual human has certain characteristics and emotion interactive ability (to identify and express emotions and affections), to endow it with artificial psychological model and the ways to identify and express emotions and affections. It is the application of artificial psychology theory in the field of virtual reality. It researches how virtual human recognizes natural human's emotion and how virtual human expresses the object's affection based on its artificial psychology model. The virtual human can study human interactive skills through communication with human, and have the same value criterions as human. Its intelligence is not the intelligence in narrow sense, but it is the intelligence in broad sense, which is not only including certain intelligence quotient, but also including certain affection quotient [5]. The virtual human uses an internal model of the agent's affective state to guide the conversational dialogue between virtual human and user.

\subsection{The Model of Virtual Human}

Traditional human machine interaction is normally based on passive instruments such as keyboard, mouse, etc. It's impossible for them to understand and express emotions. Without the ability of emotion processing, the virtual human cannot be expected to be a human-like agent in the virtual environment, and also cannot be expected to communicate with humans in a natural and harmonious way.

(1) BDI Agent Architectures

The basic BDI agent architecture designed for the virtual human is presented in Fig.1. The inputs to the agent are events from the environment and the outputs are the agent's actions. The interpreter loops and generates an action in each cycle. External and internal events are always added to an event queue. The agent's beliefs are adjusted according to those events.

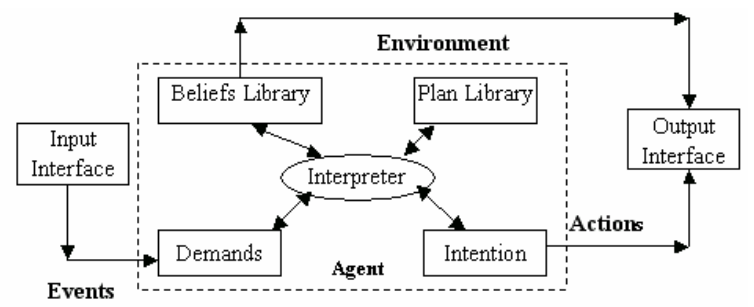

Fig. 1. This shows a basic BDI Agent Architecture

At the beginning of a cycle, plans are chosen from the plan library that specifies courses of action that may be undertaken in order to achieve the agent's goals. Next, the deliberator, a component of the interpreter, selects a subset of these plans to be adopted and adds them to the intention structure. The agent then executes one action from one plan in the intention structure. The intention and demand structures are 
modified by dropping successful goals and satisfied intentions, as well as impossible goals and unrealizable intentions. Hence, due to new external events, the agent can reactively choose to drop intended plans and/or adopt others.

(2) Affective interaction model

According to the virtual human characters, we adopted the composed structure of reactive agent and deliberative agent as the basic structure (Fig.2 shows the structure). The system structure includes two emotional information channels. The deliberative agent has affective reasoning engine for affective state recognition and modeling. The reactive agent subsystem can make reaction to the apperception results without complicated reasoning.

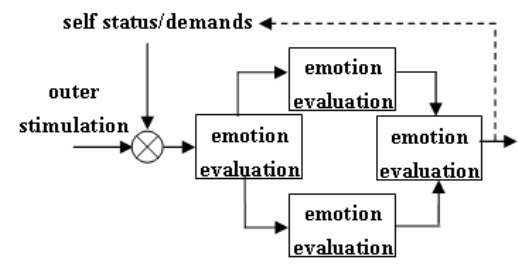

Fig. 2. The Structure of Interaction System

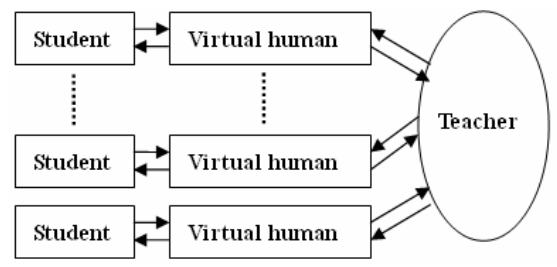

Fig. 3. The structure of the E-Learning system

\section{E-Learning System Structure}

In the e-learning process there is often one teacher for most students. To promote effectiveness of learning for the students, each student is assigned to have one virtual human. The E-Learning system structure is shown as the Fig.3. The virtual human is represented as a teacher in the e-learning platform. Except for the animate agent supplied by Microsoft Corporation, we can design a likely character or use the character from the third one. Each character has supplied a lot of interface function. It can dialog to the student face to face through the facial expression recognition system and the voice recognition system. The virtual human can change his facial expression emotion according to the learner's emotion state. Thus the e-learning process is making more and more personalized and humanized.

In the e-learning interactive system, extracting and validating emotional cues through analysis of users' facial expressions is very important. The process of the facial expression subsystem is generally carried out according to following stages: detection of the face, the automatic extraction of contours of the permanent features of the face to knowing: the eyes, the eyebrows and the lips. Extracted contours being sufficiently realistic, we then according to the changes of the facial expression character points to recognize the six universal emotions on the face (joy, surprise, fear, disgust, anger and sadness).The video frequency facial expression character point track and recognition technology is presented in reference [8]. A speech emotion recognition algorithm was developed based on the statistical and temporal features of the acoustic parameters for discriminating between emotions. The process of the speech emotion recognition subsystem is carried out in reference $[9,10]$. 


\section{Conclusion and Future Work}

This paper simply reports our exploratory approach in the teaching computation. The virtual human interaction has been used in the e-learning system in order to realize the harmonious emotion interaction. Some technologies and effective arithmetic are used in the system. This system is able to recognize the emotional state of the learner according to his facial expression. As a result, it has realized the personalized and humanized human-machine interactive function. However the virtual human interactive technology is not mature, especially the facial expression recognition rate is relatively low, so the system is only an original one. There are many aspects in the system needing to be improved. In the future we will improve and better our system, especially in facial expression track speed, recognition rate. With the development of the virtual human interaction, the virtual human will not only be used in learning in interaction ,but also capable of direct psychological enrichment tasks, such as mental therapy and entertainment.

Acknowledgments. This paper is supported by National Natural Science Foundation of China (No. 60433030) and Excellent Talent Culture Foundation of Beijing City (No.20051D0502214).

\section{References}

1. Picard R. Affective Computing. Cambridge, MA: MIT Press, 1997

2. Kinard, E. M.: Perceived and actual academic competence in maltreated children. Child Abuse and Neglect, Vol. 25, 1 (2001) 33-45

3. LeDoux, J..The emotional brain: The mysterious underpinnings of emotional life. Weiden $\neg$ feld \& Nicholson, London (1998)

4. Goleman, D.: Emotional intelligence. Bantam Books, New York (1995)

5. Weimin Xue, Zhiliang Wang, Zhehua Wei. A new method for simulating human emotions[J]. Journal of university of science and technology Beijing. Vol.10, No. 2, April. 2003,10( 2): $72-74$

6. Picard, R. W., Papert, S., Bender, W., Blumberg, B., Breazeal, C., Cavallo, D., Machover, T., Resnick, M., Roy, D., Strohecker, C.: Affective Learning - A Manifesto. BT Technology Journal Vol. 22, 4 (2004) 253-269

7. Luigi Anolli,etc The Potential of Affective Computing in E-Learning: MYSELF project experience". Proceedings of INTERACT 2005 Conference - Workshop on eLearning and Human-Computer Interaction: Exploring Design Synergies for more Effective Learning Experiences, Rome, 12-15 September 2005.

8. Weimin Xue. Facial Expression Recognition Based on Gabor Filter and SVM. Chinese Journal of Electronics [J]. October.2006, 15(4A):809 812.

9. Xue Weimin. Research on Speech Interaction of Affective Robot. Proceedings of the 7th International Conference on Electronic Measurement and Instruments, ICEMI'2005. 16-18 Aug., 2005, Beijing, China, (7) :344 347.

10. Jiang Danning, Cai Lianhong. Speech emotion recognition using acoustic features. Journal of Tsinghua University (Science and Technology).2006, 46(1):86-89 